\title{
Preface for articles from ELPUB2017
}

\author{
Leslie Chan ${ }^{\mathrm{a}}$ and Fernando Loizides ${ }^{\mathrm{b}}$ \\ ${ }^{a}$ University of Toronto Scarborough, 1265 Military Trail, Toronto, M1C1A4, Canada \\ E-mail: chan@utsc.utoronto.ca \\ ${ }^{\mathrm{b}}$ School of Computer Sciences \& Informatics, Cardiff University, 5 The Parade, Cardiff, CF24 3AA, \\ Wales, $U K$ \\ E-mail: LoizidesF@cardiff.ac.uk
}

The eight articles in this issue were first presented at the ELPUB2017 conference (International Conference on Electronic Publishing) that took place in Cyprus, June 6-8 $2017 \mathrm{http}: / / \mathrm{www}$. cyprusconferences.org/elpub2017/. The authors of these articles took advantage of the offer by IOS Press and revised their original submissions for this special issue without publication fee.

21 years ago when the ELPUB conference series first started, the term "electronic publishing" promised all manner of potential that the Web and network technologies could bring to scholarly communication, scientific research and technical innovation. Indeed, over the last two decades we have seen tremendous developments across all these domains, and at the same time our social, economical and political lives have been completely transformed.

Open Science represents one such transformation, and not surprisingly, the elements that make Open Science possible, including open access, open data, open software, and other domains of open have been regular topics presented and debated at previous ELPUB conferences.

However, development and diffusion of open research practices are highly uneven across disciplines and across regions. And despite the common claims that Open Science improves transparency and accountability throughout the research life cycle while democratizing the knowledge production process, empirical research and conceptual validation of these ideas has been limited. In addition, there is a growing tendency to conceptualize Open Science as a set of conditions waiting to be met, without regard for regional differences, including cultural and historical contexts of knowledge production.

The theme of the ELPUB2017 conference was Expanding Perspectives on Open Science: Communities, Cultures and Diversity in Concepts and Practices. It was intended to generate discussion and debate on the potential and limitations of openness. In the original Call for Papers, we invited researchers and practitioners from diverse backgrounds to share their results and ideas towards a highly interactive forum.

We also asked potential presenters to consider exploring alternative models of interaction and cocreation between scholars and citizen scientists, and the role of dissemination and publishing within these interactions. To stimulate submissions, we included these questions in the open call: Who determines the agenda and direction of emerging discourses around Open Science? How does Open Science challenge the current positions and power of players and agents in varying institutional contexts? Are we seeing a converging global view of Open Science, or are there disciplinary, regional, and other differences that are important to consider? What are the gaps between existing Open Science policies, regulatory frameworks, and implementation requirements and how should they be addressed? How do 
Open Science agendas relate to the Open Innovation agendas of governments, funders and institutions? What is the impact of these agendas on research funding and dissemination practices?

By assessing these interlinked questions, the aim was to improve our understanding of current challenges and opportunities in the ecosystem of open science, and how to move forward collaboratively in developing an inclusive system that works for a much broader range of participants.

In all, a total of 26 research and practitioner papers and 7 posters were presented at this year's conference, along with 4 workshops on the first day of the conference. The papers were published in a Proceedings published by IOS Press http://ebooks.iospress.nl/book/expanding-perspectives-on-open-sciencecommunities-cultures-and-diversity-in-concepts-and-practices-proceedings-of-the-21st-internationalconference-on-electron. The papers represent a broad range of topics related to Open Science, from provision of common infrastructure, innovative tools, new publishing models, sustainability models, and policy provisions. We also have a broad range of conceptual papers exploring the boundaries and diversities of open research practices in varying institutional and cultural contexts.

The eight papers in this issue also reflect the diversity of perspectives and approaches. In addition, four of the papers are authored by members of the Open and Collaborative Science in Development Network (OCSDNet http://www.ocsdnet.org), and the authors come from countries including Jamaica, Brazil, Peru, Colombia, and Argentina. These authors presented results and reflections on their respective research on the promises and challenges of open research collaboration, adding to a growing body of understanding of diverse regional and local conditions that enable or hinder openness.

We would like to take this opportunity to extend an invitation to all of you to the 22st edition of the conference, which will be held in Toronto, Canada. The theme is Connecting the Knowledge Commons: From Projects to Sustainable Infrastructure. We hope to see an even more diverse group of presenters, topics, and attendees at this conference, and look forward to welcoming you to Toronto! 